\title{
Nonlinear evolution of torsional Alfvén waves
}

\author{
S. Vasheghani Farahani ${ }^{1}$, V. M. Nakariakov ${ }^{2,3}$, E. Verwichte ${ }^{2}$, and T. Van Doorsselaere ${ }^{1,4}$ \\ ${ }^{1}$ Centre for mathematical Plasma Astrophysics, Department of Mathematics, KU Leuven, Celestijnenlaan 200B bus 2400, \\ 3001 Leuven, Belgium \\ e-mail: soheil .vasheghanifarahani@wis . kuleuven . be \\ ${ }^{2}$ Centre for Fusion, Space and Astrophysics, Physics Department, University of Warwick, Coventry CV4 7AL, UK \\ 3 Central Astronomical Observatory of the Russian Academy of Sciences at Pulkovo, 196140 St Petersburg, Russia \\ ${ }^{4}$ Postdoctoral fellow of the FWO vlaanderen
}

Received 9 May 2012 / Accepted 24 July 2012

\section{ABSTRACT}

\begin{abstract}
Aims. We study the efficiency of the energy transfer to shorter scales in the field-aligned direction - the parallel nonlinear cascade that accompanies the propagation of torsional Alfvén waves along open magnetic fields in the solar and stellar coronae, and compare it with the same effects for the shear Alfvén wave. The evolution of the torsional Alfvén wave is caused by the back reaction of nonlinearly induced compressive perturbations on the Alfvén wave.

Methods. The evolution of upwardly propagating torsional Alfvén waves is considered in terms of the second-order thin flux-tube approximation in a straight untwisted and non-rotating magnetic flux-tube. The Cohen-Kulsrud equation for weakly nonlinear torsional waves is derived. In the model, the effect of the cubic nonlinearity on the propagation of long-wavelength axisymmetric torsional waves is compared with the similar effect that accompanies the propagation of plane linearly-polarised (shear) Alfvén waves of small amplitude.

Results. The solution to the Cohen-Kulsrud type equation for torsional waves shows that their evolution is independent of the plasma$\beta$, which is in contrast to the shear Alfvén wave. In a finite- $\beta$ plasma, the nonlinear evolution of torsional Alfvén waves is slower and the parallel nonlinear cascade is less efficient than those of shear Alfvén waves. These results have important implications for the analysis of possible heating of the plasma and its acceleration in the upper layers of solar and stellar coronae. In particular, onedimensional models of coronal heating and wave acceleration, which use shear Alfvén waves instead of torsional Alfvén waves, over-estimate the efficiency of these processes.
\end{abstract}

Key words. magnetohydrodynamics (MHD) - waves - Sun: corona - Sun: activity - magnetic fields

\section{Introduction}

Torsional Alfvén waves are (linearly incompressive) perturbations that do not perturb the plasma density and are a type of magnetohydrodynamic (MHD) wave in structured plasmas (Van Doorsselaere et al. 2008b) that travel at the Alfvén speed along the magnetic field. These waves are azimuthal perturbations of the plasma that are linked to the azimuthal component of the magnetic field (Vasheghani Farahani et al. 2010). Alfvén waves are important as they may play a role in coronal heating (e.g. Hollweg et al. 1982; Ofman 2005) and both solar and stellar wind acceleration (e.g. Cranmer 2009; Charbonneau \& MacGregor 1995; Suzuki 2007).

There is only indirect observational evidence of torsional Alfvén waves in the solar corona. Zaqarashvili (2003) suggested that standing torsional Alfvén waves may be observed in coronal loops from the periodic variation in the spectral line widths. Banerjee et al. (2009) reported the possible presence of propagating torsional Alfvén waves in polar coronal holes, as manifested by the non-thermal broadening of emission lines. A similar interpretation can be made for the nonthermal broadening of coronal lines observed during an eclipse (Singh et al. 2011). Torsional Alfvén waves were also proposed as one possible explanation of the transverse motions of coronal rain plasmas observed by Hinode/SOT (Antolin \& Verwichte 2011). Jess et al. (2009) reported the possible detection of torsional Alfvén waves in the chromosphere. In addition,
Tapping (1983) and Grechnev et al. (2003) linked the observed periodic behaviour of the microwave emission generated in solar flares with the possible existence of torsional waves in flaring magnetic flux tubes. However, the unequivocal identification of torsional waves in solar atmospheric structures requires the simultaneous analysis of the Doppler and magnetic signals. Torsional Alfvén waves should not be confused with the kink modes of coronal plasma structures (see the discussion in Van Doorsselaere et al. 2008a; Van Doorsselaere et al. 2008b).

A series of theoretical studies of coronal heating and solar wind acceleration by Alfvén waves were carried out in onedimensional (1D) models by Suzuki (2004), Suzuki \& Inutsuka (2005), Verdini \& Velli (2007), Suzuki (2007, 2008, 2011), and Verdini et al. (2012). A key ingredient of these models is the nonlinear cascade of the wave energy from the low-frequency injection range to shorter scales, where it becomes subject to dissipation by MHD and kinetic mechanisms. In Alfvén waves, this effect becomes possible because of the nonlinear interaction of the waves with the nonlinearly induced compressive perturbations, or, in other words, the self-interaction of Alfvén waves. For weakly-nonlinear Alfvén waves, this process is described by the Cohen-Kulsrud evolutionary equation (Cohen \& Kulsrud 1974). In particular, Nakariakov et al. (2000b) derived a generalised Cohen-Kulsrud equation for linearly-polarised spherical Alfvén waves in coronal holes, and demonstrated that the nonlinear transfer of energy to shorter scales can play a significant role in the conditions typical of the solar and stellar coronae. 
However, in the solar and stellar coronal context, the consideration of torsional Alfvén waves is more appropriate than the shear Alfvén wave, as the existence of shear Alfvén waves would require the horizontal extent of wave fronts to be much larger than their vertical wavelength. Thus, comparison of the nonlinear evolution of torsional and shear Alfvén waves is an important issue that is needed for the justification of the 1D models of coronal heating and solar wind acceleration or for their modification.

Vasheghani Farahani et al. (2011) studied weakly nonlinear long-wavelength torsional Alfvén waves in magnetic flux tubes of the solar corona. They considered a straight magnetic flux tube and used the second-order thin flux-tube approximation (Zhugzhda 1996). Compressive perturbations induced nonlinearly by long-wavelength axisymmetric torsional waves were found to oscillate at twice the frequency of the source waves themselves. In contrast to shear Alfvén waves, the plasma- $\beta$ was found to have no effect on the efficiency of the generation of compressive perturbations in propagating torsional Alfvén waves. The reason for this discrepancy is that the natural speed of the longitudinal compressive perturbations in a magnetic flux is the tube (or cusp) speed, while in a uniform medium it is the sound speed. The MHD tube speed is always lower than both the sound and Alfvén speeds.

In this paper, we consider the back reaction of the nonlinearly-induced compressive perturbations on torsional Alfvén waves. The Cohen-Kulsrud evolutionary equation for torsional waves is derived, and its solution is compared with the corresponding solution obtained for shear Alfvén waves (Verwichte et al. 1999). The efficiency of the nonlinear parallel cascade of energy in torsional and shear Alfvén waves is compared with the use of spectral methods.

\section{Self-interaction of torsional waves by compressive perturbations}

We consider a static and straight magnetic flux tube of cylindrical cross-section and radius $R$ that has an axial equilibrium magnetic field $B_{z 0}$, a mass density $\rho_{0}$, a pressure $p_{0}$, and a cross-sectional area $A_{0}$. The internal total pressure is balanced by the external total pressure $p_{\mathrm{T} 0}^{\text {ext }}$. In the following, our analysis is carried out in cylindrical coordinates $(r, \varphi, z)$. Linear torsional waves are azimuthal perturbations of the equilibrium, with the vorticity $\Omega=v_{\varphi} / r$ being accompanied by the alternating magnetic twist (electric current density) $J=B_{\varphi} / r$. We restrict our attention to the consideration of long-wavelength (relative to the tube radius $R$ ) axisymmetric perturbations. At the axis of the flux tube $r=0$, the magnetic twist and the azimuthal velocity are zero, and the description of the torsional waves requires the second-order thin flux-tube approximation (Zhugzhda 1996) instead of the first order approximation (Roberts \& Webb 1978). In that approximation, perturbations of physical quantities are Taylor-expanded with respect to the radial coordinate, and asymptotically analysed using a small parameter equal to the ratio of the tube radius to the characteristic length scale of the perturbations in the longitudinal direction. In the second-order thin flux-tube approximation, the perturbations of the azimuthal and radial components of the magnetic field and the plasma velocity increase linearly with the radial coordinate. In addition, the Alfvén speed inside the tube is assumed to be constant, hence the effects of Alfvén wave phase-mixing are absent. Substituting the perturbations of the physical parameters in to the MHD equations, keeping the linear terms on the left hand side and gathering the nonlinear terms on the right hand side (RHS), we obtain

$\frac{\partial \Omega}{\partial t}-\frac{B_{z 0}}{4 \pi \rho_{0}} \frac{\partial J}{\partial z}=N_{1}$,

$\rho_{0} \frac{\partial u}{\partial t}+\frac{\partial p}{\partial z}=N_{2}$

$\frac{\partial \rho}{\partial t}+\rho_{0} \frac{\partial u}{\partial z}+2 \rho_{0} V=0$

$\frac{\partial J}{\partial t}-B_{z 0} \frac{\partial \Omega}{\partial z}=N_{4}$,

$\frac{\partial B_{z}}{\partial t}+2 B_{z 0} V=0$

$\frac{\partial p}{\partial t}-C_{\mathrm{s}}^{2} \frac{\partial \rho}{\partial t}=0$

$p+\frac{1}{4 \pi} B_{z 0} B_{z}-\frac{A_{0} \rho_{0}}{2 \pi} \frac{\partial V}{\partial t}-\frac{A_{0} B_{z 0}}{16 \pi^{2}} \frac{\partial^{2} B_{z}}{\partial z^{2}}=N_{7}$,

where $C_{\mathrm{s}}^{2}=\gamma p_{0} / \rho_{0}$ is the sound speed, $u$ is the velocity perturbation along the cylinder axis and $V$ is the radial derivative of the radial velocity $v_{r}$ (see Vasheghani Farahani et al. 2011, for details). In the following, we assume that in the system there are initially no compressive perturbations. (A similar assumption is made for the standard derivation of the Cohen-Kulrsrud equation, see Cohen \& Kulsrud 1974.) Taking into account the quadratic nonlinear terms of $\Omega$ and $J$ and retaining the nonlinearity caused by the back reaction of the twist on the compressive variables, the RHS of Eqs. (1)-(7) are expressed as

$$
\begin{aligned}
N_{1}= & -\frac{J}{4 \pi \rho_{0}} \frac{\partial B_{z}}{\partial z}+\frac{B_{z 0}}{4 \pi \rho_{0}}\left[\frac{B_{z}}{B_{z 0}}-\frac{\rho}{\rho_{0}}\right] \frac{\partial J}{\partial z} \\
& -2 V \Omega-u \frac{\partial \Omega}{\partial z}, \\
N_{2}= & -\frac{R^{2}}{4 \pi} J \frac{\partial J}{\partial z}, \\
N_{4}= & -\frac{\partial(u J)}{\partial z}+B_{z} \frac{\partial \Omega}{\partial z}-2 V J, \\
N_{7}= & -\frac{A_{0} \rho_{0}}{2 \pi} \Omega^{2}+\frac{A_{0}}{8 \pi^{2}} J^{2}+p_{\mathrm{T}}^{\mathrm{ext}},
\end{aligned}
$$

where $p_{\mathrm{T}}^{\text {ext }}$ is the total pressure in the external medium. The other nonlinear terms are zero. The induced compressive perturbations may be written in terms of $J$ with the use of Eqs. (2), (3), and (5)-(7)

$$
\begin{aligned}
& \frac{\partial u}{\partial t}=-\frac{C_{\mathrm{s}}^{2}}{\rho_{0}} \frac{\partial \rho}{\partial z}-\frac{R^{2}}{4 \pi \rho_{0}} J \frac{\partial J}{\partial z}, \\
& \frac{\partial V}{\partial t}=-\frac{1}{2 \rho_{0}} \mathcal{D}_{\mathrm{s}} \rho+\frac{R^{2}}{8 \pi \rho_{0}} \frac{\partial}{\partial z}\left(J \frac{\partial J}{\partial z}\right), \\
& \frac{\partial^{2} B_{z}}{\partial t^{2}}=\frac{B_{z 0}}{\rho_{0}} \mathcal{D}_{\mathrm{s}} \rho-\frac{R^{2} B_{z 0}}{4 \pi \rho_{0}} \frac{\partial}{\partial z}\left(J \frac{\partial J}{\partial z}\right),
\end{aligned}
$$

hence

$$
\begin{array}{r}
\left(C_{\mathrm{A}}^{2}+C_{\mathrm{s}}^{2}\right) \mathcal{D}_{\mathrm{T}} \rho+\frac{A_{0}}{4 \pi} \mathcal{D}_{\mathrm{A}} \mathcal{D}_{\mathrm{s}} \rho=\frac{A_{0}}{2 \pi} \frac{\partial^{2}}{\partial t^{2}}\left(\frac{J^{2}}{4 \pi}-\rho_{0} \Omega^{2}\right) \\
+\frac{R^{2}}{4 \pi}\left[C_{\mathrm{A}}^{2}+\frac{A_{0}}{4 \pi} \mathcal{D}_{\mathrm{A}}\right] \frac{\partial}{\partial z}\left(J \frac{\partial J}{\partial z}\right),
\end{array}
$$

where $C_{\mathrm{A}}=B_{z 0} / \sqrt{4 \pi \rho_{0}}$ is the Alfvén speed, and

$\mathcal{D}_{\mathrm{T}, \mathrm{s}, \mathrm{A}}=\frac{\partial^{2}}{\partial t^{2}}-C_{\mathrm{T}, \mathrm{s}, \mathrm{A}}^{2} \frac{\partial^{2}}{\partial z^{2}}$, and $C_{\mathrm{T}}^{2}=\frac{C_{\mathrm{A}}^{2} C_{\mathrm{s}}^{2}}{C_{\mathrm{A}}^{2}+C_{\mathrm{s}}^{2}}$. 
We note that the perturbation of the total pressure in the external medium, $p_{\mathrm{T}}^{\text {ext }}$, is ignored because we concentrate only on the compressive perturbations inside the flux tube, and weakly nonlinear torsional waves do not perturb the flux-tube boundary (Vasheghani Farahani et al. 2011). The first term on the RHS of Eq. (15) corresponds to the magnetic tension and centrifugal forces, and the second term to the ponderomotive force.

The torsional Alfvén wave equation is then obtained by eliminating $\Omega$ from Eqs. (1) and (4)

$\mathcal{D}_{\mathrm{A}} J=B_{z 0} \frac{\partial N_{1}}{\partial z}+\frac{\partial N_{4}}{\partial t}$.

We note that the terms with the product $J(\partial J / \partial z)$ are related to the ponderomotive force, which causes the nonlinear selfinteraction of Alfvén waves that we are interested in.

Considering weakly nonlinear waves, we may change to the running frame of reference

$\xi=z-C_{\mathrm{A}} t, \quad \tau=t$.

Assuming that the perturbations are slowly varying, we rewrite Eqs. (12)-(15) in the new frame of reference as

$u=\frac{\left(C_{\mathrm{A}}^{2}+C_{\mathrm{s}}^{2}\right) R^{2}}{8 \pi \rho_{0} C_{\mathrm{A}}^{3}} J^{2}, \quad V=-\frac{C_{\mathrm{S}}^{2} R^{2}}{16 \pi \rho_{0} C_{\mathrm{A}}^{3}} \frac{\partial J^{2}}{\partial \xi}$,

$B_{z}=-\frac{B_{z 0} C_{\mathrm{s}}^{2} R^{2}}{8 \pi \rho_{0} C_{\mathrm{A}}^{4}} J^{2}, \quad \rho=\frac{R^{2}}{8 \pi C_{\mathrm{A}}^{2}} J^{2}$.

In addition, in the thin flux-tube approximation, since the torsional wave exists on any magnetic surface as long as a linear dependence on $r$ is satisfied, the linear relation between $J$ and $\Omega$ is obtained from the wave equation by combining Eqs. (1) and (4) in the linear regime. We obtain

$\Omega=-\frac{C_{\mathrm{A}}}{B_{z 0}} J$.

In the new frame of reference, Eq. (17) is re-written with the use of Eqs. (19), (20) as

$\frac{\partial J}{\partial \tau}+\frac{3 R^{2}}{16 \pi \rho_{0} C_{\mathrm{A}}} J^{2} \frac{\partial J}{\partial \xi}=0$,

with the solution

$\frac{B_{\varphi}}{B_{z 0}}=a f\left(\frac{\xi}{L}-\frac{3}{4} \frac{C_{\mathrm{A}}}{L}\left(\frac{B_{\varphi}}{B_{z 0}}\right)^{2} \tau\right)$,

where $a$ is a dimensionless constant, $L$ is a characteristic lengthscale, and the relation $J=B_{\varphi} / R$ has been used. We note that the function $f$ denotes the initial shape of the torsional wave, at the time $\tau=0$.

Figure 1 shows the evolution of an initially harmonic Alfvén wave, plotting the value $B_{\varphi} / B_{z 0}$ VS. the running coordinate $\xi / L$, using Eq. (22). One can see that the shape is gradually distorted and the wave shape departs from the harmonic. Moreover, in contrast to the typical quadratically nonlinear evolution in terms of the Burgers equation describing, e.g. slow magnetoacoustic waves in coronal loops and polar plumes (see Nakariakov et al. 2000c; Ofman et al. 2000), the Alfvén wave shape differs from the saw-tooth. The nonlinear steepening occurs twice as frequently as in the case of the Burgers equation. It is a typical feature of the waves affected by cubic nonlinearity, in particular Alfvén waves (e.g. Verwichte et al. 1999; Nakariakov et al. 2000b).

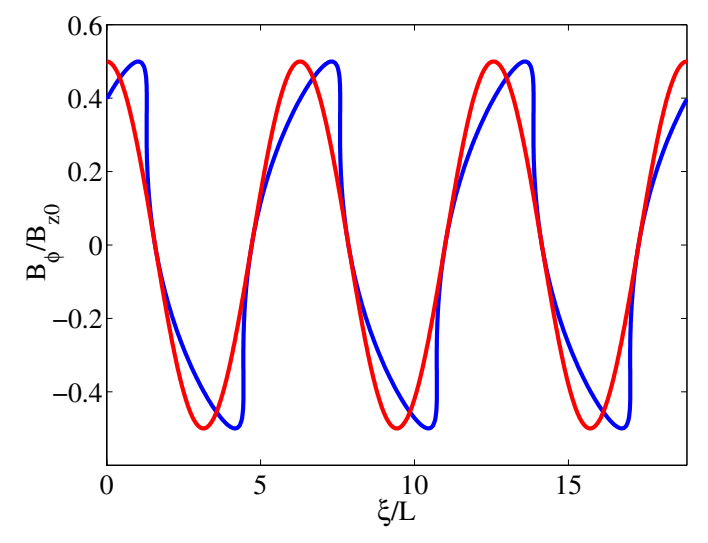

Fig. 1. Evolution of an initially harmonic, weakly nonlinear, torsional Alfvén wave. $B_{\varphi} / B_{z 0}$ is plotted against the distance along the flux tube $\xi / L$, normalised to the initial wavelength. The red curve is the initial shape at $\bar{\tau}=\tau C_{\mathrm{A}} / L=0$. The blue curve corresponds to the time $\bar{\tau}=5.5$ when the wave is about to overturn.

\section{Nonlinear cascade in torsional and shear Alfvén waves}

We compare Eq. (21) to its equivalent for a plane, shear Alfvén wave. We consider linearly polarised Alfvén waves for which $v_{y}$ and $B_{y}$ propagate in the $z$-direction with no dependence on the $x$ and $y$ coordinates $(\partial / \partial y=0$ and $\partial / \partial x=0)$. Following the formalism developed in Verwichte et al. (1999) and Nakariakov et al. (2000a), the equivalent of Eq. (21) is

$\frac{\partial B_{y}}{\partial \tau}+\frac{3 C_{\mathrm{A}}}{16 \pi \rho_{0}\left(C_{\mathrm{A}}^{2}-C_{\mathrm{s}}^{2}\right)} B_{y}^{2} \frac{\partial B_{y}}{\partial \xi}=0$,

with the solution

$\frac{B_{y}}{B_{z 0}}=b f\left(\frac{\xi}{L}-\frac{3}{4} \frac{C_{\mathrm{A}}^{3}}{L\left(C_{\mathrm{A}}^{2}-C_{\mathrm{s}}^{2}\right)}\left(\frac{B_{y}}{B_{z 0}}\right)^{2} \tau\right)$,

where $b$ is a dimensionless constant. Since $J=B_{\varphi} / R$, Eqs. (21) and (23) appear to be similar, but interestingly differ in the coefficients of the nonlinear terms. In the zero- $\beta$ limit, the coefficients become identical and the solutions of both Eqs. (21) and (23) coincide. However, the discrepancy appears in the finite- $\beta$ case, when the torsional wave solution in Eq. (22) shows no dependence on the sound speed (or the value of $\beta$ ), while the shear wave solution in Eq. (24) has a singularity in the case of the resonance between the phase speed of the wave, Alfvén speed, and the sound speed.

The evolution of both the torsional (Eq. (22)) and shear (Eq. (24)) Alfvén pulses of an initially Gaussian shape is shown in Fig. 2 for different values of the plasma- $\beta$. In all cases, the pulse experiences nonlinear steepening, resulting in a weak shock. In the zero- $\beta$ case, torsional and shear Alfvén pulses evolve identically. With the increase in the plasma- $\beta$ (defined here as $C_{\mathrm{s}}^{2} / C_{\mathrm{A}}^{2}$ ), the nonlinear distortion of the shear Alfvén pulse starts earlier and the shock forms quicker. Moreover, for $\beta>1$, the nonlinear distortion experienced by the torsional and shear pulses differ considerably: in the shear pulse, the shock appears at the backward slope, while in the torsional shock it appears at the front slope, as in the $\beta<1$ case.

To quantify the efficiency of the generation of higher harmonics in the cases of torsional and shear Alfvén pulses, we show their power spectra in Fig. 3. It is evident that the plasma- $\beta$ 

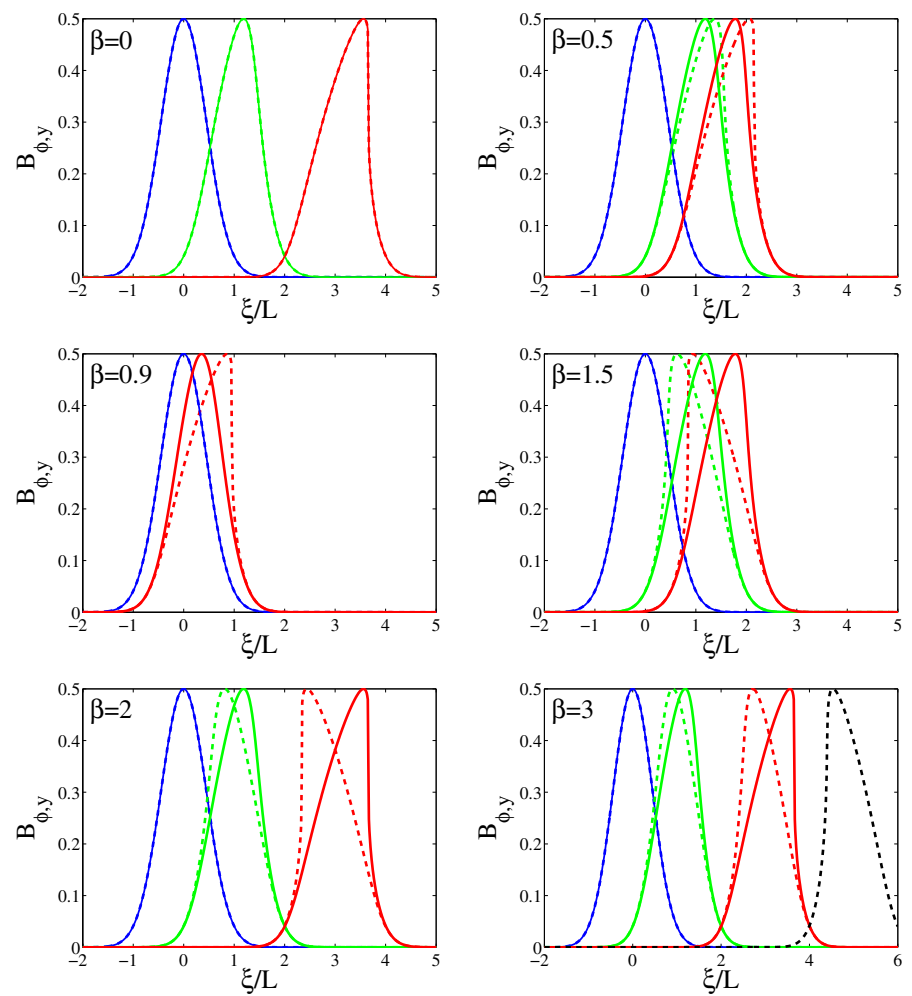

Fig. 2. Snapshots of a Gaussian profile using Eq. (22) for the torsional Alfvén wave (solid lines), and Eq. (24) for the shear Alfvén wave (dashed lines), where $B_{\varphi} / B_{z 0}$ and $B_{y} / B_{z 0}$ are plotted against the running coordinate $\xi / L$ normalised to the initial length of the pulse. The value of the plasma- $\beta$ is stated on each panel and the characteristic time is $\bar{\tau}=\tau C_{\mathrm{A}} / L$. The top, left panel represents the times $\bar{\tau}=0,1,3$ and the top, right panel the times $\bar{\tau}=0,1,1.5$. The middle left panel is for the times $\bar{\tau}=0,0.3$, and the middle right panel the times $\bar{\tau}=0,1,1.5$. The bottom left panel is for the times $\bar{\tau}=0,1,3$, and the bottom right panel the times at $\bar{\tau}=0,1,3,4$.

does not affect the nonlinear cascade in torsional Alfvén waves, in contrast to the case for the shear Alfvén waves.

\section{Conclusions}

We have studied the nonlinear self-interaction of longwavelength torsional Alfvén waves of weak amplitude, in a straight, untwisted, and non-rotating magnetic flux tube. This is caused by the interaction of the waves with the nonlinearly induced, compressive, longitudinal perturbations. Comparison is made with the same phenomenon for shear (plane) Alfvén waves. We can summarise our findings as follows:

1. Nonlinear evolution of torsional Alfvén waves is described by the Cohen-Kulsrud equation, as for shear Alfvén waves. However, in contrast to the case of shear waves, the coefficient in the nonlinear term is independent of the plasma- $\beta$.

2. In the cases of both shear and torsional Alfvén waves, the nonlinear self-interaction causes a steepening of the waves. The nonlinear evolution of the torsional and shear waves is identical in the zero- $\beta$ limit, while in the case of finite- $\beta$ the nonlinear steepening of torsional waves develops slower than that of the shear waves. Moreover, in the hot plasma where the sound speed is greater than the Alfvén speed, shocks are formed at the opposite slopes in torsional and shear Alfvén waves.
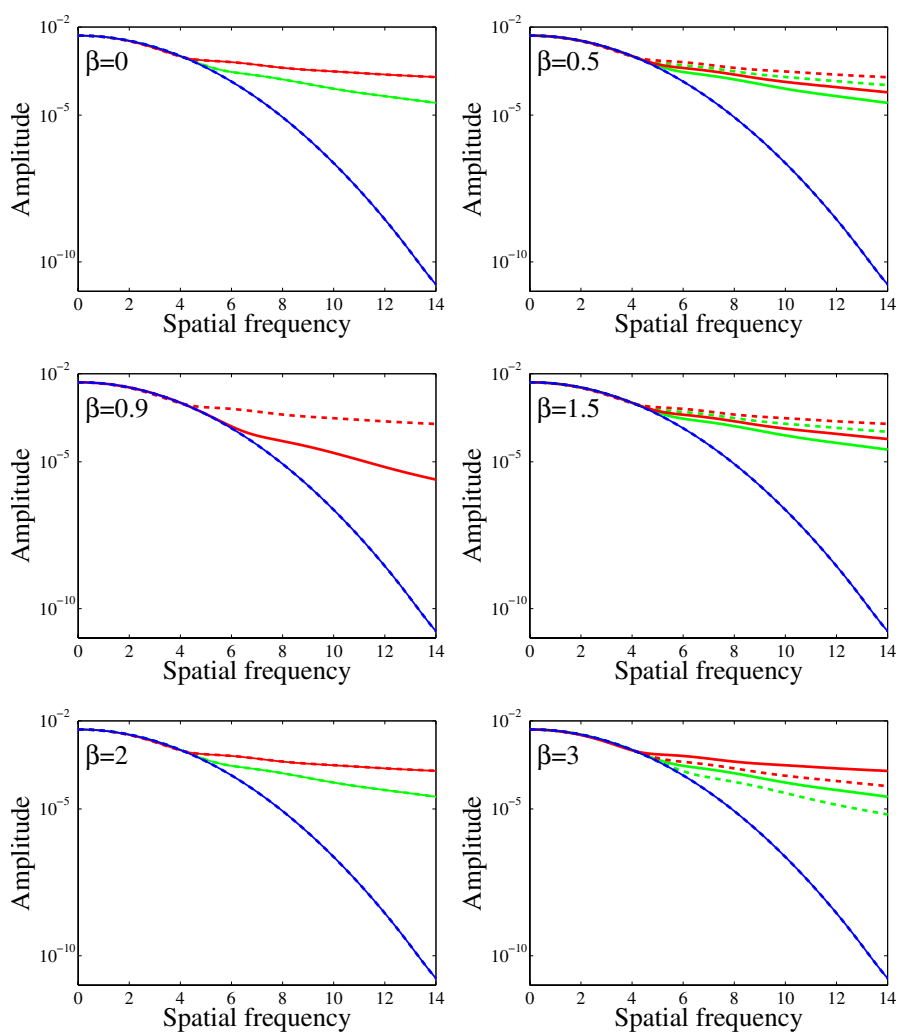

Fig. 3. Spatial spectra's of the torsional (solid lines) and shear (dashed lines) Alfvén profiles shown in Fig. 2. The times are the same as for the corresponding panels.

3. Spatial spectra of torsional and shear Alfvén waves show that the nonlinear flattening of the spectrum in the case of torsional waves is less efficient than for shear waves in the finite- $\beta$ case.

Our study shows that in a finite- $\beta$ plasma, the nonlinear evolution of torsional Alfvén waves is slower than that of shear Alfvén waves. Hence, the parallel nonlinear cascade that is the transfer of energy to smaller parallel wave numbers in the case of torsional waves is less efficient than for shear waves. This result has important implications for the analysis of the wave-based plasma heating and acceleration mechanisms in the upper layers of solar and stellar coronae. In particular, 1D models of coronal heating and wave acceleration, which use shear Alfvén waves instead of torsional Alfvén waves, overestimate the efficiency of these processes and need to be revised.

Acknowledgements. T.V.D. acknowledges the funding of an FWO Odysseus grant. S.V.F. acknowledges the funding of the GOA/2009-009 of the KU leuven Research Council.

\section{References}

Antolin, P., \& Verwichte, E. 2011, ApJ, 736, 121

Banerjee, D., Pérez-Suárez, D., \& Doyle, J. G. 2009, A\&A, 501, L15

Charbonneau, P., \& MacGregor, K. B. 1995, ApJ, 454, 901

Cohen, R. H., \& Kulsrud, R. M. 1974, Phys. Fluids, 17, 2215

Cranmer, S. R. 2009, Liv. Rev. Sol. Phys., 6, 3

Grechnev, V. V., White, S. M., \& Kundu, M. R. 2003, ApJ, 588, 1163

Hollweg, J. V., Jackson, S., \& Galloway, D. 1982, Sol. Phys., 75, 35 
S. Vasheghani Farahani et al.: Nonlinear evolution of torsional Alfvén waves

Jess, D. B., Mathioudakis, M., Erdélyi, R., et al. 2009, Science, 323, 1582 Nakariakov, V. M., Mendoza-Briceño, C. A., \& Ibáñez S., M. H. 2000a, ApJ, 528,767

Nakariakov, V. M., Ofman, L., \& Arber, T. D. 2000b, A\&A, 353, 741

Nakariakov, V. M., Verwichte, E., Berghmans, D., \& Robbrecht, E. 2000c, A\&A, 362,1151

Ofman, L. 2005, Space Sci. Rev., 120, 67

Ofman, L., Nakariakov, V. M., \& Sehgal, N. 2000, ApJ, 533, 1071

Roberts, B., \& Webb, A. R. 1978, Sol. Phys., 56, 5

Singh, J., Hasan, S. S., Gupta, G. R., Nagaraju, K., \& Banerjee, D. 2011, Sol. Phys., 270, 213

Suzuki, T. K. 2004, MNRAS, 349, 1227

Suzuki, T. K. 2007, ApJ, 659, 1592

Suzuki, T. K. 2008, Nonlinear Proc. Geophys., 15, 295

Suzuki, T. K. 2011, Space Sci. Rev., 158, 339
Suzuki, T. K., \& Inutsuka, S. 2005, ApJ, 632, L49

Tapping, K. F. 1983, Sol. Phys., 87, 177

Van Doorsselaere, T., Brady, C. S., Verwichte, E., \& Nakariakov, V. M. 2008a, A\&A, 491, L9

Van Doorsselaere, T., Nakariakov, V. M., \& Verwichte, E. 2008b, ApJ, 676, L73 Vasheghani Farahani, S., Nakariakov, V. M., \& Van Doorsselaere, T. 2010, A\&A, 517, A29

Vasheghani Farahani, S., Nakariakov, V. M., van Doorsselaere, T., \& Verwichte, E. 2011, A\&A, 526, A80

Verdini, A., \& Velli, M. 2007, ApJ, 662, 669

Verdini, A., Grappin, R., \& Velli, M. 2012, A\&A, 538, A70

Verwichte, E., Nakariakov, V. M., \& Longbottom, A. W. 1999, J. Plasma Phys., 62,219

Zaqarashvili, T. V. 2003, A\&A, 399, L15

Zhugzhda, Y. D. 1996, Phys. Plasmas, 3, 10 